\title{
Treatment Process for Neuropathy Involving Science-Based Clinical Research and Medical Technology Combinations
}

\author{
Bao Thai \\ Advanced Nerve \& Health Center (ANHC), Carrollton, TX, USA \\ Email: admin@dfwnerveandhealth.com
}

How to cite this paper: Thai, B. (2021) Treatment Process for Neuropathy Involving Science-Based Clinical Research and Medical Technology Combinations. World Journal of Neuroscience, 11, 267-278.

https://doi.org/10.4236/wjns.2021.114019

Received: June 24, 2021

Accepted: September 12, 2021

Published: September 15, 2021

Copyright $\odot 2021$ by author(s) and Scientific Research Publishing Inc. This work is licensed under the Creative Commons Attribution International License (CC BY 4.0).

http://creativecommons.org/licenses/by/4.0/

\begin{abstract}
In this study, we will demonstrate the ability of multiple and specific alternative medical technologies used in combination that support the body's natural ability to repair peripheral nerves in neuropathy patients. This paper looks to review currently known biological foundations for the regenerative effects of Low-Level Laser Therapy, Pulsed Electromagnetic Field Therapy, Infrared and Near Infrared Therapy, and Nutritional Protocols to demonstrate the significant potential to repair peripheral nerve injuries. There is favorable evidence to expand functional recovery in diverse types of peripheral nerve injuries. The conclusion from this study indicates using a specific combination of technologies that scientifically enhances the body's ability to repair nerves is more effective in helping patients with neuropathies than the normal standard of care.
\end{abstract}

\section{Keywords}

Neuropathy, Class IV Laser, Diabetic Neuropathy, PEMF, NeuroLight, LLLT

\section{Introduction}

Current standard of care treatment options for all neuropathies is to suppress neuropathic symptoms. These options are unfortunately mildly successful due to the further decline in the patient's health relative to their nerve function and as well as their ability to perform daily tasks. All treatments that are widely used are mainly designed to decrease nociceptive transmission [1]. In this study, we have treated 500 patients. Our measure of success is the percentage of improvement in the patient's condition reported by the patient as well as functional outcomes such as sleep quality, reduction in symptomologies, increase in physical function, increase in sensation, decrease in pain, and reduction of neuropathy medications. 


\section{Research Study Background}

$90 \%$ of our patients in this study have been to other doctors ranging from their primary care physician to specialists such as neurologists, podiatrists, physical therapists, chiropractors, and pain management MD's. Most of these patients have also had diagnostic studies and been diagnosed with neuropathy by numerous providers. Their treatment at these facilities have ranged from nerve decompression surgeries, implantation of spinal stimulators, ablation of nerves, lidocaine injections, steroid injections, electrical stimulation, and medications all of which proved to be unsuccessful.

In our treatment of neuropathy patients, we have seen $96 \%$ of our patients report at least $50 \%$ or more improvement in symptomologies. Over $85 \%$ reduced their intake of anticonvulsants, antidepressants, and or pain medication prescribed for their neuropathy. Over 78\% reported better sleep quality. $85 \%$ of the patients reported that their balance and gait saw positive improvement.

\section{Study Conclusion}

The conclusion of this study is that using a specific combination of technologies that scientifically enhances the body's ability to repair nerves is more effective in helping patients with neuropathies than the normal standard of care. Furthermore, the use of this process is far safer for patients compared to medications and surgeries; (of which can have negative consequences) (cite a study that shows how bad surgeries and medications are) as well as achieving sustainability as long as the patients maintain a healthy active lifestyle.

\section{Previous Methods of Neuropathy Treatment}

The standard level of care by most medical physicians is to prescribe medication. Treatment goals include improving pain control, however, there is little research on the overall treatment plan once the patient has reached their peak of dosage allowed. The main classes of agents used to treat diabetic peripheral neuropathic pain include tricyclic antidepressants, anticonvulsants, serotonin-norepinephrine reuptake inhibitors, opiates, and opiate-like substances, and topical medications.

\subsection{Statistic Review}

As you can see from the data (Table 1, Table 2), the current treatment options for neuropathy come with numerous side effects and still not accounting for the progression of the disease while on medications.

The main clinical debate has always been, "Can peripheral nerves repair and regenerate themselves? Are they able to restore their functionality?" This has been an ongoing debate among physicians and medical professionals. If you review and understand the science; then the answer is unequivocally "yes". Peripheral nerves can repair and regenerate. There have been numerous studies that show the ability of peripheral nerves to regenerate themselves following a transection. The process is broken down into two main parts. The process is a com- 
plex multicellular response that guides and sustains axonal regrowth [3] [4]. While difficult, it is possible.

Table 1. Drug interactions in treatment for diabetic peripheral neuropathic pain.

\begin{tabular}{|c|c|}
\hline \multirow{5}{*}{$\begin{array}{l}\text { Carbamazepine } \\
\text { (Tegretol) }\end{array}$} & May increase toxic effects of CNS depressants* \\
\hline & $\begin{array}{l}\text { May decrease effectiveness of CYP1A2 substrates } \dagger, \text { CYP2B6 substrates } \ddagger, \text { CYP2C9 substrates, CYP3A4 substrates } \| \text {, oral } \\
\text { contraceptives, thyroid medications, and TCAs }\end{array}$ \\
\hline & Effects can be decreased by CYP3A4 inducers \\
\hline & Toxic effects can be increased by CNS depressants* and SSRIs \\
\hline & Absolutely contraindicated with MAOIs \\
\hline \multirow{6}{*}{$\begin{array}{l}\text { Duloxetine } \\
\text { (Cymbalta) }\end{array}$} & May increase toxic effects of CNS depressants*, \\
\hline & SSRIs, and warfarin (Coumadin) \\
\hline & May decrease effectiveness of TCAs \\
\hline & Effects can be decreased by CYP1A2 inducers ${ }^{* *}$ \\
\hline & Toxic effects can be increased by CNS depressants* and SSRIs \\
\hline & Absolutely contraindicated with MAOIs \\
\hline \multirow{3}{*}{$\begin{array}{l}\text { Oxycodone, } \\
\text { controlled release } \\
\text { (Oxycontin) and } \\
\text { morphine }\end{array}$} & May increase toxic effects of CNS depressants*, MAOIs, and SSRIs \\
\hline & Effects can be decreased by CYP3A4 inducers \\
\hline & Toxic effects can be increased by CNS depressants ${ }^{\star}$ \\
\hline \multirow{2}{*}{$\begin{array}{l}\text { Pregabalin (Lyrica) } \\
\text { and gabapentin } \\
\text { (Neurontin) }\end{array}$} & May increase toxic effects of CNS depressants* \\
\hline & Toxic effects can be increased by CNS depressants* \\
\hline \multirow{2}{*}{ Tramadol (Ultram) } & May increase toxic effects of CNS depressants* and SSRIs \\
\hline & Toxic effects can be increased by CNS depressants*, SSRIs, and TCAs \\
\hline \multirow{4}{*}{ TCAs } & $\begin{array}{l}\text { May increase toxic effects of CNS depressants*, QTc-prolonging agents } \dagger \dagger \text {, SSRIs, St. John's wort, sulfonylureas, tramadol, } \\
\text { and warfarin }\end{array}$ \\
\hline & Effects can be decreased by carbamazepine and St. John's wort \\
\hline & Toxic effects can be increased by CNS depressants*, duloxetine, QTc-prolonging agents $\dagger \dagger$, SSRIs, and St. John’s wort \\
\hline & Absolutely contraindicated with MAOIs \\
\hline \multirow{4}{*}{$\begin{array}{l}\text { Venlafaxine } \\
\text { (Effexor) }\end{array}$} & May increase toxic effects of CNS depressants ${ }^{\star}$ and SSRIs \\
\hline & Effects can be decreased by CYP3A4 inducers \\
\hline & Toxic effects can be increased by CNS depressants ${ }^{\star}$, CYP2D6 inhibitors $\ddagger$, CYP3A4 inhibitors $₫ \S$, and SSRIs \\
\hline & Absolutely contraindicated with MAOIs \\
\hline
\end{tabular}

CNS = central nervous system; CYP = cytochrome P450; MAOIs = monoamine oxidase inhibitors; SSRIs = selective serotonin reuptake inhibitors; TCAs = tricyclic antidepressants. ${ }^{*}$ Carbamazepine, gabapentin, opiates, pregabalin, tramadol, trazodone, and TCAs. $\dagger-$ Duloxetine, estrogens, flutamide, mirtazapine (Remeron), propranolol, and ropinirole (Requip). $¥-$ Bupropion (Wellbutrin), cyclophosphamide, efavirenz (Sustiva), promethazine, and selegiline (Eldepryl).\$-Celecoxib (Celebrex), dapsone, fluoxetine (Prozac), fosphenytoin (Cerebyx), glimepiride (Amaryl), glipizide (Glucotrol), losartan (Cozaar), monte-lukast (Singulair), phenytoin (Dilantin), tamoxifen, trimethoprim/sulfamethoxazole (Bactrim, Septra), trimethoprim, and warfarin. I-Alfuzosin (Uroxatral), amiodarone (Cordarone), atorvastatin (Lipitor), calcitriol (Rocaltrol), citalopram (Celexa), clonazepam (Klonopin), enalapril (Vasotec), estrogens, felodipine, ketoconazole, progesterones, and tetracycline. - Carbamazepine, dexamethasone, efavirenz, fosphenytoin, phenytoin, and

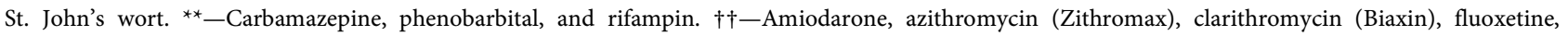
haloperidol (formerly Haldol), risperidone (Risperdal), and sotalol (Betapace). desipramine (Norpramin), duloxetine, haloperidol, imipramine (Tofranil), isoniazid, ketoconazole, lidocaine (Xylocaine), methadone, pioglitazone (Actos), and sertraline (Zoloft). $\$ \$-$ Amiodarone, cimetidine, clotrimazole (Lotrimin), cyclosporine (Sandimmune), desipramine, diltiazem (Cardizem), efavirenz, erythromycin, fluconazole (Diflucan), grapefruit juice, haloperidol, metronidazole (oral [Flagyl], gel [Metrogel]), sertraline, tetracycline, and verapamil. Information from reference [2]. 
Table 2. Common adverse effects from treatment for diabetic peripheral neuropathic pain.

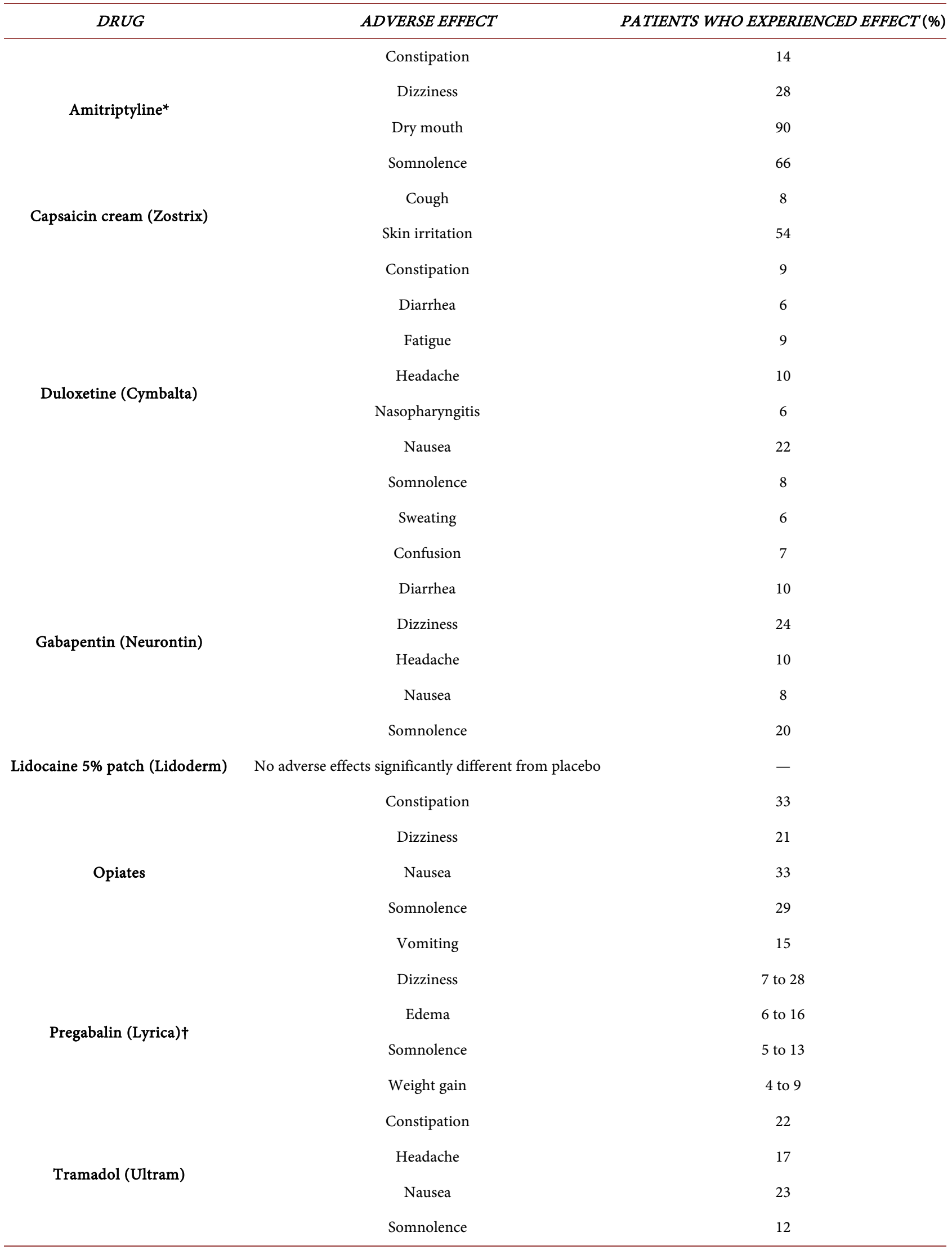




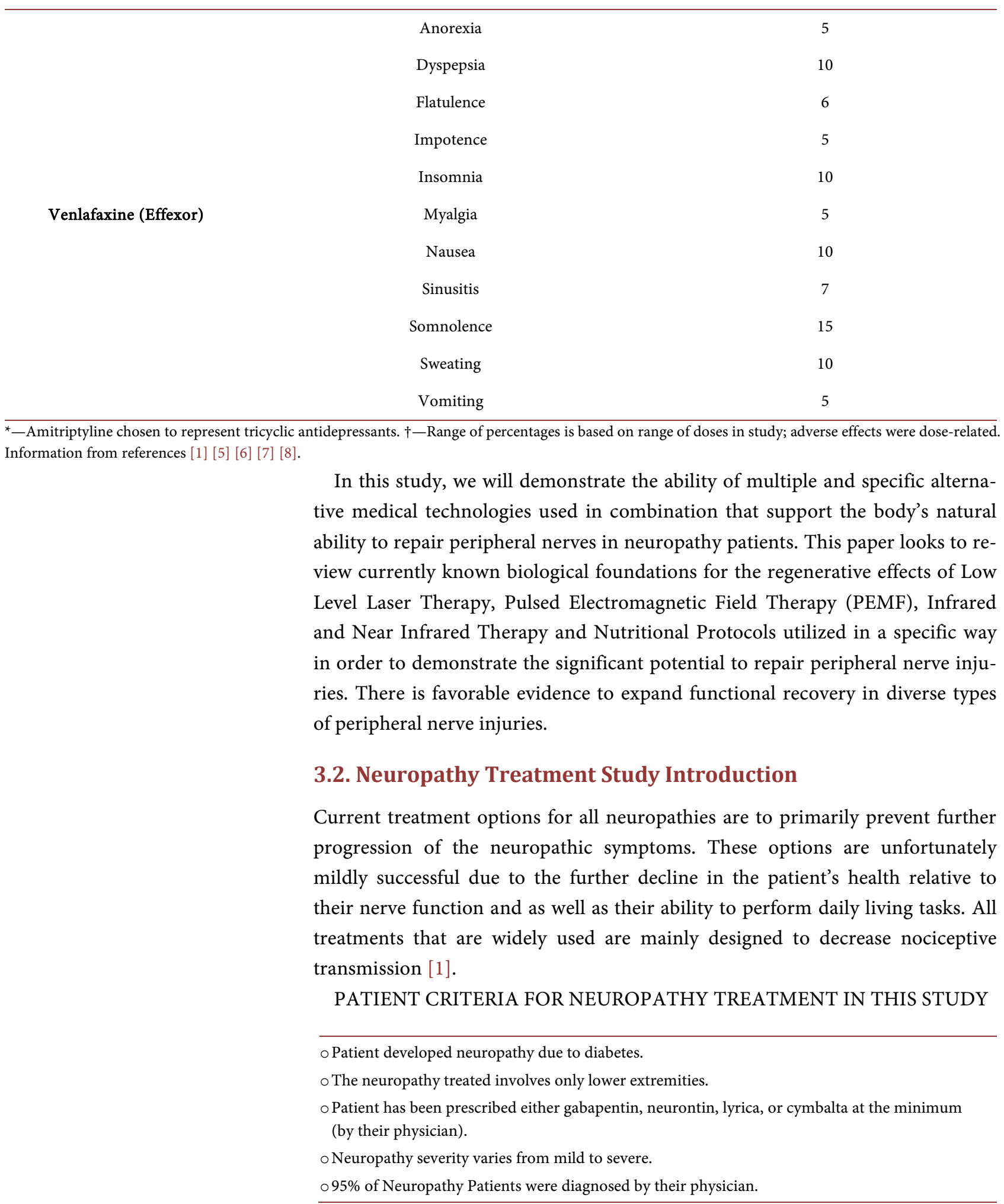

In this study we have treated over 2000 patients successfully. Our measure of success is the percentage of improvement in the patient's condition reported by the patient as well as functional outcomes such as sleep quality, reduction in 
symptomologies, increase in physical function, increase in sensation, decrease in pain, and reduction of neuropathy medications.

Neuropathy is one of the major medical conditions on the rise.

Doctors are seeing more and more patients come in with neuropathic symptoms that range from sensory symptoms (e.g., numbness, tingling), weakness, autonomic symptoms (e.g., early satiety, impotence, orthostatic hypertension, sweat abnormalities), or neuropathic (burning, stabbing, electrical) pain [1]. The causes of neuropathies can range from diabetes, toxicity, trauma, and idiopathic.

Most of our patients developed neuropathy as a result of diabetes. The remainder of the patients in this study developed neuropathy either as having multiple causes or due to platinum-based chemotherapy, idiopathic, or surgically induced. All the patients in the study presented with numbness, pain, and tingling of their feet and legs. Some had issues with the upper extremities as well, but we did not consider that in our study. All these patients were prescribed either gabapentin, neurontin, lyrica, or cymbalta at the minimum (by their physician).

The severity of the patients in the study varied from mild to severe with most of the patients in the moderate to severe category. Most of these patients also reported that balance and sleep were an issue as well that interfered with their quality of life. Patients were evaluated by reviewing a detailed medical history and previous diagnostic testing such as Electromyography (EMG) or Nerve Conduction Velocity (NCV) tests. Over 95\% of the patients have all been diagnosed with neuropathy by a medical doctor prior to becoming a patient at our clinic.

\subsection{Patient Demographics}

Patients in the study all suffered some form of neuropathy. The breakdown is as follows for patient demographics.

Gender: Female Patients: $53 \%$ Male Patients: $47 \%$
Exercise percentage-25\% worked out at least $2 \mathrm{x}$ a week
Alcohol-60\% consumed 2 drinks or more per week
Smoking-10\% smoked on a regular basis
On other prescription meds - $94 \%$
Nutrition-75\% reported that their nutrition was not good
Comorbidity-80\% suffered from other health problems
Retired-60\%
Non Retired-40\%
Age Percentage
$50-60-37 \%$
$60-70-53 \%$
$70-80$ - $8 \%$
$80-2 \%$


The patients in this study all suffered with neuropathic symptoms in their legs and feet. Majority patients reported feeling the sensation of "walking on something." The common descriptions were walking on cardboard and or springs. Most symptoms were located at the balls of the feet.

$80 \%$ of the patients had bilateral symptoms with neither the right or the left worse.

$60 \%$ of the patients reported that their sleep was being affected.

$95 \%$ of those patients either could not sleep through the night or their quality of sleep was negatively affected.

$85 \%$ said that the medications they were on did not relieve their symptoms completely. Only $30 \%$ of the patients said that they felt that meds were really helping.

$70 \%$ of the patients said they could not say that the medications were doing anything to help them. That they were just taking it because their doctor told them too.

$70 \%$ of the patients suffered gait issues and reported that their ability to walk was being affected. $100 \%$ of the patients reported concern that if their neuropathy is not treated or controlled, the severity of progression could lead to requiring a cane, wheelchair, and/or potentially permanent nerve damage for example.

Upon initial examination the detailed medical history was performed by the doctor to determine the following:

- Potential causes of neuropathy;

- Length of time patient has experienced neuropathy symptoms;

- Progression of disease;

- Changes/Adjustments to their daily life since diagnosed with neuropathy;

- Medication history;

- Surgical history;

- Previous neuropathy treatments with outcome details;

- Visual examination.

Once the vital information was obtained and a foundation was established, one of the following or all these tests were performed depending on cause and previous diagnostic testing:

- Vibration testing;

- Sensory testing.

$\circ$ Gait analysis.

The testing utilized would be determined by the symptoms the patient currently presents or experiencing. The purpose would be to determine the severity of the neuropathy. The next step in the process was to determine the changeability factor of the neuropathy. Through my research and treating thousands of patients over time, we discovered a diagnostic process that has a direct correlation between potential success or potential failure of our specialized, detailed and personalized neuropathy treatment.

\subsection{Neuropathy Patient Trial Examination Method}

The trial testing consists of the following medical technologies;

- Class IV 60-watt laser.

- Pulsed Electromagnetic Field (PEMF) (settings determined by causation). 
- Neurolight therapy.

Patients who underwent the testing were all determined to be symptomatic. The patient symptoms were recorded and agreed upon by the doctor and patient. Motor and sensory functions of the nerve were to be evaluated.

The consulting doctor would go over the medical history with a medical technician. The treatment areas and specific treatment settings were discussed before the diagnostic treatment. The medical technician would explain the diagnostic process to the patient and then transport the patient to the treatment area(s).

The patient would be seated in our treatment room. The medical technician would ask a series of questions as well as give instruction to the patient.

- Patient is to remove shoes and socks if applicable.

- The patient's feet would then be sanitized thoroughly.

- A clear plastic bag is placed over the patient's foot up to the knee.

- The Neurolight Therapy Unit would be placed on the patient's feet from the calf to the bottom of the foot wrapped to the anterior foot.

- The PEMF Unit would be placed with the larger of the 2 pads wrapped around the inferior part of the patient's feet. The polarity of the pads would be noted as going from inferior to superior. PEMF setting(s) vary for this device and we can change the frequency, wavelength, and or power. These changes are designed to increase mitochondrial function, increase circulation, and decrease inflammation [9].

○ Treatment time would be 20 mins total unless otherwise noted.

- The patient would be instructed to monitor their sensation and any changes.

- Once the treatment was completed the patient was unhooked from the machines and then escorted by a medical technician into another treatment room.

- The patient is placed in a prone position. The clear bags were removed.

- Class IV Laser treatment time would be 5 mins per leg. The breakdown per leg is as follows: (The medical technician would administer safety glasses for all persons in the room.)

- $1 \mathrm{~min}$ on the lateral side of the leg concentrating on the peroneal nerve.

- 2 mins on the anterior foot and ankle.

- 2 mins on the posterior foot.

- The medical technician is moving the Class IV laser at 1 inch per second and keeping a distance of less than 1 inch.

- During the treatment process, the medical technician is constantly asking the patient if they can feel where he/she is using the Class IV Laser. This is to determine sensation sensitivity and proprioception.

- Same process is administered to the anterior and posterior foot in the same manner. The total amount of energy administered per area is 4500 Joules.

Once the trial treatment process is complete, the patient is asked to move and walk on their feet to determine if changes to neuropathy symptoms. The consulting doctor records and analyzes the results to determine the changeability factor. In all of the patients we have seen in the past, the ones that do not see or 
feel a change do not respond to further treatment.

In my research and trial testing, we have concluded that most patients will see positive and sustainable results with 18 - 33 treatments (consisting of 2 - 3 treatment appointments weekly). The potential outcomes for all patients accepted into care is achieving 50\% improvement in their condition. On the patient's first appointment, $\mathrm{He} / \mathrm{She}$ is provided a detailed plan for their neuropathy treatment, which includes home care instruction and specific nutritional guidelines to follow. Each patient receives a NeuroLight Unit to use daily at home to continue the treatment process when not in the clinic.

The nutritional side to our plan is an anti-inflammatory and/or keto food plan. The goal is to limit the carbohydrate and sugar intake. The patient mainly is to concentrate on healthy proteins and vegetables.

As other studies have demonstrated; inflammation from sugars and carbohydrates will cause neuropathies to progress [10]. In our experience, the patients that follow the nutritional component show greater positive changes in their neuropathy. In the patients that are slow to respond to the nutrition guidelines, we find they are not seeing/feeling the results as quickly. By not following nutritional guidelines, I am referring to the continued intake of carbohydrates and/or sugars (consuming sodas, etc.).

\section{Overall Neuropathy Treatment Goals}

- Increase nerve growth factors (NGF);

- Increase protein synthesis of GAP 43;

○ Increase blood flow.

In our clinic we use a GaAlAs Class IV laser at a minimum of 30watts and a maximum of 60 watts. Laser treatment is performed by a trained medical technician. The distance of treatment from diode to the skin is not to exceed 2 inches. The more distance that is created will cause the loss of power, and the beams will be reflected off instead of being absorbed by the photoreceptors. The dual wavelength GaAlAs $(810 \mathrm{~nm}), \mathrm{GaAl}(980 \mathrm{~nm})$ of our lasers are $810 \mathrm{~nm}$ to $980 \mathrm{~nm}$. These have been proven to cause the increase in activation of schwann cells, stem cells, and nerve growth factors as indicated in numerous studies [11]. Nerve cells respond particularly well to LLLT, which has been proposed for a range of neurostimulation and neuromodulation applications, and recent progress in neural stimulation and regeneration [12]. Significant acceleration of revascularization and angiogenesis of the peripheral nerve has been documented [13]. Furthermore, a reduction of hemorrhages and increase in blood supply happens with laser therapy. Wallerian degeneration decreased while higher axonal density occurs as well [13]. Schwann cells are the myelinating cell of the peripheral nervous system. Its basic function is to help create myelin. This makes them the very foundation of nerve repair. Activation of schwann cells has been demonstrated by numerous studies using laser therapy at $810 \mathrm{~nm}$. The lasers power and wavelength are a big determining factor on the success of schwann 
cell activation.

Our Neurolight therapy is using near infrared and infrared therapy to increase vasodilation of an area thereby causing an increase in blood flow. [14] Next, patients will also undergo our PEMF therapy. PEMF therapy through many scientific studies indicated that it can promote nerve regeneration and could be used for the treatment of neuropathic pain [10]. PEMF therapy was originally discovered and used by orthopedic surgeons to help bone growth. Through published studies it shows that PEMF at the cellular level activates mitochondrial function. This leads to further production of osteoblasts and other proteins for growth or repair [15]-[24].

Patients in this group all had some form of myalgia as well causing their symptoms to get worse. To address this part of their issue we utilize a device called the Rapid Release. This device is placed on the patient's skin. It vibrates at a high frequency causing microcirculation to occur. In addition, it causes relaxation of the muscles involved. This in turn helps to increase blood flow and decrease inflammation in those specific areas.

\section{Conclusions}

The results of this study are as follows:

○ $98 \%$ of the patients reported $50 \%$ or more improvement.

○ $76 \%$ of the patients reported $80 \%$ more improvement.

Results patients reported are as follows:

- Reduction or elimination of pain.

○ Reduction or elimination of numbness.

- Reduction or elimination of tingling.

- Improvement of balance.

- Increase stamina and ability to walk.

- Increase in happiness.

The results the patients reported were progressive in nature. As treatment continued, they noticed a decrease in symptoms and an increase in functionality. Most patients expressed their concern of being on medications. They were advised to talk to their prescribing doctors to re-evaluate the need seeing how they were not experiencing the neuropathy symptoms.

Patients that continued a healthy lifestyle; limiting the number of carbohydrates, sugars, and refined foods reported that their outcomes are still consistent without treatment. Our patients also reported that $98 \%$ are either completely or mostly of their neuropathy medications as well.

This study demonstrates that the use of medical technologies with specialized equipment, specific settings, time of treatment, nutritional protocols, and home therapy as well can be more effective in helping patients with neuropathy, then the current standard of care. It is to note that not all equipment is the same and there must be a certain amount of knowledge that is needed to be able to achieve these results. The process that is used is purely based on previous science and 
studies. I have taken that knowledge and the clinical outcomes I have seen in treating many patients and combined what has worked. The result of our study is that my process is more effective in helping patients with neuropathic symptoms vs medications and surgery. Considering the research of the equipment we use; it is safe to conclude that activation of Schwann cells which leads to repair of myelin has occurred in the patients of the study.

\section{Conflicts of Interest}

The author declares no conflicts of interest regarding the publication of this paper.

\section{References}

[1] Watson, J.C. and Dyck, P.J. (2015) Peripheral Neuropathy: A Practical Approach to Diagnosis and Symptom Management. Mayo Clinic Proceedings, 90, 940-951.

[2] Lexi-Comp (Online Reference Library) (2009) American Pharmaceutical Association Hudson, Ohio. http://online.lexi.com

[3] Cattin, A.L. and Lloyd, A.C. (2016) The Multicellular Complexity of Peripheral Nerve Regeneration. Current Opinion in Neurobiology, 39, 38-46.

[4] Tonge, D.A., Aaronson, O.S., Golding, J.P. and Jaggers, D. (1996) Cellular Migration and Axonal Outgrowth from Adult Mammalian Peripheral Nerves in vitro. Journal of Neurobiology, 29, 151-64.

[5] Eisenberg, E., McNicol, E. and Carr, D.B. (2006) Opioids for Neuropathic Pain. Cochrane Database of Systematic Reviews, Article ID: CD006146.

[6] Hollingshead, J., Dühmke, R.M. and Cornblath, D.R. (2006) Tramadol for Neuropathic Pain. Cochrane Database of Systematic Reviews, Article ID: CD003726.

[7] Meier, T., Wasner, G., Faust, M., et al. (2003) Efficacy of Lidocaine Patch 5\% in the Treatment of Focal Peripheral Neuropathic Pain Syndromes: A Randomized, Double-Blind, Placebo-Controlled Study. Pain, 106, 151-158.

[8] Max, M.B., Culnane, M., Schafer, S.C., et al. (1987) Amitriptyline Relieves Diabetic Neuropathy Pain in Patients with Normal or Depressed Mood. Neurology, 37, 589-596. https://doi.org/10.1212/WNL.37.4.589

[9] Ross, C.L., Ang, D.C. and Almeida-Porada, G. (2019) Targeting Mesenchymal Stromal Cells/Pericytes (MSCs) With Pulsed Electromagnetic Field (PEMF) Has the Potential to Treat Rheumatoid Arthritis. Frontiers in Immunology, 10, 266.

[10] Rowin, J. (2019) Integrative Neuromuscular Medicine: Neuropathy and Neuropathic Pain: Consider the Alternatives. Muscle Nerve, 60, 124-136. https://doi.org/10.1002/mus.26510

[11] Ginani, F., Soares, D.M., Barreto, M.P. and Barboza, C.A. (2015) Effect of LowLevel Laser Therapy on Mesenchymal Stem Cell Proliferation: A Systematic Review. Lasers in Medical Science, 30, 2189-2194.

[12] Tsai, S.R. and Hamblin, M.R. (2017) Biological Effects and Medical Applications of Infrared Radiation. Journal of Photochemistry and Photobiology B, 170, 197-207.

[13] Mashhoudi Barez, M., Tajziehchi, M., Heidari, M.H., Bushehri, A., Moayer, F., Mansouri, N., Safavi Naini, N. and Movafagh, A. (2017) Stimulation Effect of Low Level Laser Therapy on Sciatic Nerve Regeneration in Rat. Journal of Lasers in Medical Sciences, 8, S32-S37. 
[14] Wan, Q., Yang, S., Li, L. and Chu, F. (2017) Effects of Far Infrared Therapy on Arteriovenous Fistulas in Hemodialysis Patients: A Meta-Analysis. Renal Failure, 39, 613-622.

[15] Patterson, T.E., Sakai, Y., Grabiner, M.D., et al. (2006) Exposure of Murine Cells to Pulsed Elec-Tromagnetic Fields Rapidly Activates the mTOR-Signaling Pathway. Bioelectromagnetics, 27, 535-544.

[16] Selvamurugan, N., Kwok, S., Vasilov, A., Jefcoat, S.C. and Partridge, N.C. (2007) Effects of BMP-2 and Pulsed Electromagnetic Field (PEMF) on Rat Primary Osteoblastic Cell Proliferation and Gene Expression. Journal of Orthopaedic Research, 25, 1213-1220. https://doi.org/10.1002/jor.20409

[17] Midura, R.J., Ibiwoye, M.O., Powell, K.A., et al. (2005) Pulsed Electromagnetic Field Treatments Enhance the Healing of Fibular Osteotomies. Journal of Orthopaedic Research, 23, 1035-1046. https://doi.org/10.1016/j.orthres.2005.03.015

[18] Garland, D.E., Moses, B. and Salver, W. (1991) Fracture Healing: Long-Term Follow-Up of Fracture Nonunions Treated with PEMFs. Contemporary Orthopaedics, 22, 295-302.

[19] Simmons, J.W., Mooney, V. and Thacker, I. (2004) Pseudarthrosis after Lumbar Spine Fusion: Non-Operative Salvage with Pulsed Electromagnetic Fields. American Journal of Orthopedics, 33, 27-30.

[20] Mooney, V. (1990) A Randomized Double-Blind Prospective Study of the Efficacy of Pulsed Electromagnetic Fields of Interbody Lumbar Fusions. Spine, 15, 708-712.

[21] Foley, K., et al. (2008) Randomized, Prospective, and Controlled Clinical Trial of Pulsed Electromagnetic Field Stimulation for Cervical Fusion. The Spine Journal, 8, 436-442.

[22] Zborowski, M., Androjna, C., Waldorff, E.L. and Midura, R.J. (2015) Comparison or Therapeutic Magnetic Stimulation with Electric Stimulation of Spinal Column Vertebrae. IEEE Transactions on Magnetics, 51, Article ID: 5001009. https://doi.org/10.1109/TMAG.2015.2458297

[23] Schnoke, M. and Midura, R.J. (2007) Pulsed Electromagnetic Fields Rapidly Modulate Intracellular Signaling Events in Osteoblastic Cells: Comparison to Parathyroid Hormone and Insulin. Journal of Orthopaedic Research, 25, 933-940. https://doi.org/10.1002/jor.20373

[24] Ibiwoye, M.O., Powell, K.A. and Grabiner, M.D. (2004) Bone Mass is Preserved in a Criticalsized Osteotomy by Low Energy Pulsed Electromagnetic Fields as Quantitated by in vivo Microcomputed Tomography. Journal of Orthopaedic Research, 22, 1086-1093. https://doi.org/10.1016/j.orthres.2003.12.017 